\title{
Tipping Points in der Rechtsentwicklung: Zur Situation von Low-Budget Musiker:innen im Spannungsfeld von Verwertungsinteresse und Drittnutzung
}

\author{
Sophie Beaucamp", Simon Schrör"
}

\section{Einleitung}

Die gegenwärtigen Entwicklungen im Europäischen Urheberrecht machen einmal mehr deutlich, dass mit den digitalen Möglichkeiten des Schaffens und Veröffentlichens von Musik, Videos, Texten und anderen Formen des kreativen Ausdrucks das Urheberrecht von einem branchenspezifischen zu einem alltagsrelevanten Rechtsgebiet geworden ist. Fragen der rechtssicheren Nutzung von bestehendem Material stellen sich nicht nur professionellen Künstler:innen oder der Medienindustrie, sondern vor allem durch den Austausch auf Plattformen ${ }^{1}$ auch Privatpersonen und Kreativen, die zum Beispiel fremdreferenzielle Musik schaffen und veröffentlichen. Gleichzeitig sind die Grenzen zwischen Berufs- und Hobbymusiker:innen und zwischen gewerblich und nicht-gewerblich Agierenden nicht leicht zu bestimmen. Verdienstverhältnisse sind fluide und reine Privatnutzung ist bei einem potenziell globalen Publikum schwer zu bestimmen oder zu planen.

Während die professionelle Musikindustrie mit dem Sample-Clearing eine Möglichkeit gefunden zu haben scheint, Sampling durch individuelle Vertragsabschlüsse einigermaßen rechtssicher zu gestalten, bleibt die Situa-

* Sophie Beaucamp, LL.M. (LSE) ist Volljuristin und arbeitet als wissenschaftliche Mitarbeiterin am Weizenbaum-Institut in der Forschungsgruppe Vertrauen in verteilten Umgebungen. Ihr Forschungsschwerpunkt liegt auf dem Gebiet des Rechts des geistigen Eigentums sowie des Informationstechnologierechts.

** Simon Schrör, (M.A) ist Soziologe und Leiter der Forschungsgruppe Verlagerungen in der Normsetzung am Weizenbaum-Institut. Forschungsschwerpunkte sind die Rolle immaterieller Güter bei der Entwicklung von Kultur und Wirtschaft sowie sozio-normative Verlagerungsprozesse im Zuge der Digitalisierung.

1 Der Begriff ,Plattform' wird in diesem Beitrag verwendet im Sinne von Diensteanbietern für das Teilen von Online-Inhalten entsprechend der Richtlinie (EU) 2019/790 des Europäischen Parlaments und des Rates über das Urheberrecht und die verwandten Schutzrechte im digitalen Binnenmarkt vom 17. April 2019. 
tion in Gänze doch unklar und prekär. Das Feld der fremdreferenziell Arbeitenden beschränkt sich eben nicht nur auf Berufsmusiker:innen. Neben frei von jedem Verdienstinteresse musizierenden Privatpersonen gibt es besonders im Hip-Hop ${ }^{2}$ eine dritte Akteur:innengruppe: Low-Budget Musiker:innen, die am strukturellen Tipping Point zwischen Hobbymusik mit geringem Verwertungsinteresse und gewerblicher Musikproduktion zu verorten sind.

Die Untersuchung dieser Gruppe eignet sich analytisch besonders, um die rechtlichen Freiheiten und Grenzen, die bei fremdreferenzieller Musik von großer Wirkmächtigkeit sind, auszuloten. Am Tipping Point der Gewerblichkeit lassen sich die Logiken und Anforderungen der professionellen sowie der hobbyhaften Musikproduktion zugleich illustrieren.

In diesem Beitrag werden vor dem Hintergrund aktueller Entwicklungen in Rechtsprechung und Rechtsetzung die Spannungen untersucht, die zwischen der kulturellen Notwendigkeit des Sampelns, der unsicheren Rechtslage und dem exkludierenden System ${ }^{3}$ des professionellen SampleClearings auftreten. Anhand dieser Analyse wird schließlich gezeigt, dass das rechtliche System anders als das der Kunst oder der Wirtschaft an dieser Stelle eine funktionale und politisch gut beeinflussbare Stellschraube darstellt. Wenn sie mit Bedacht und mit Blick auf das multisystemische Gleichgewicht von Wirtschaft, Kunst und Recht vorgenommen werden, verheißen Anpassungen der rechtlichen Rahmung des Samplingregimes einen Ausweg aus der exkludierenden und bisweilen dysfunktionalen Gemengelage, die sich heute beim Soundsampling findet.

\section{Soundsampling als allgemeine Kulturtechnik im Hip-Hop}

Sampling kann definiert werden als die direkte Nutzung von kurzen, digital kopierten Ton- oder Musikstücken aus Werken Dritter. ${ }^{4}$ Besonders ver-

2 Sampling spielt insbesondere auch in elektronischer Musik eine Rolle. Hinsichtlich des Low-Budget Sektors lassen sich einige der strukturellen Überlegungen potenziell übertragen. Exemplarisch und aufgrund der kulturellen Genrespezifika konzentriert sich dieser Artikel jedoch auf das Genre des Hip-Hop.

3 Wie unter 2.2 zu zeigen sein wird.

4 Sampling kann auch nicht-digital etwa durch das Abspielen einer Schallplatte oder eines Tonbandes im Hintergrund einer Tonaufnahme stattfinden. In den Frühzeiten des Hip-Hops war dies gang und gäbe (vgl. Mikos, Interpolation and sampling, in: Androutsopoulos, HipHop, $65 \mathrm{f}$.). Heute findet Sampling jedoch fast ausschließlich digital statt (vgl. Stange-Elbe/Bronner, Musikinstrumentenindustrie, in: 
breitet ist Sampling im Hip-Hop und bei elektronischer Musik. Sampling als direkte Form der Fremdreferenzialität ist im Hip-Hop bereits in der Entstehung des Genres angelegt, ${ }^{5}$ und kann als eine "Standardtechnologie der Musikproduktion" ${ }^{6}$ begriffen werden. Weder aus hochprofessionellen, noch aus amateurhaften oder semi-professionellen Produktionen ist Sampling als Form des Aufgreifens von und des Auseinandersetzens mit Bestehendem - sei es auf inhaltlicher oder auf klanglicher Ebene - wegzudenken. ${ }^{7}$

Erkennt man Sampling als eine kulturelle Anforderung des Genres an, so wird die Frage relevant, ob, wie und von wem diese Kulturtechnik unter den realen Bedingungen und Strukturen des Musikmarktes überhaupt angewandt werden kann. Um dieser Frage nachzugehen, konzentriert sich die Betrachtung wie beschrieben auf eine Akteur:innengruppe, die am strukturellen Tipping Point ${ }^{8}$ zwischen gewerblicher und nicht gewerblicher Musikproduktion agiert: Musiker:innen im Low-Budget Hip-Hop.

\subsection{Low-Budget Hip-Hop}

Unter Akteur:innen im Low-Budget Sektor können Musiker:innen verstanden werden, die Musikprodukte schaffen und verwerten, dabei aber nicht in die Labelstrukturen der professionell organisierten Musikindustrie eingebettet sind. Zugleich verfügen die Akteur:innen dieses Sektors über äußerst begrenzte finanzielle Mittel, was wiederum maßgeblich zu deren Sonderstellung beiträgt. So können zum Beispiel physische Tonträger nicht oder nur in geringem Umfang hergestellt und vertrieben und an-

Stöckler/Tschmuck, Musikrezeption, Musikdistribution und Musikproduktion, 323).

5 Mikos, Interpolation and sampling, in: Androutsopoulos, HipHop, 66.

$6 \mathrm{Kawohl/Kretschmer,} \mathrm{Von} \mathrm{Tondichtern} \mathrm{und} \mathrm{DJs,} \mathrm{in:} \mathrm{Hofmann,} \mathrm{Wissen} \mathrm{und} \mathrm{Eigen-}$ tum, 204.

7 Das Genre des Hip-Hop setzt hier ebenso wie kontemporäre elektronische Musik die der westlichen Musikgeschichte inhärente Fremdreferenzialität fort (vgl. Carroll U. Cin. L. Rev. 2004, 1405). Dies geschieht aufgrund der bei der Entstehung des Genres vorhandenen technischen Möglichkeiten direkt und heute zumeist digital. Die damit verbundenen Haftungsfragen treten beispielsweise in der Klassik, die ebenfalls oft fremdreferenziell ist, aufgrund der nur mittelbaren Übernahme nicht auf, vgl. Arewa N.C. L. Rev. 2006, 547, 601ff.

8 Wie gezeigt wird, ist dieser Kipppunkt keinesfalls als dichotome Grenze zu verstehen. Fälle können zwischen gewerblichen und nicht-gewerblichen Logiken oszillieren, oder sich im Zeitverlauf unintendiert wandeln. 
waltliche Beratung nicht oder nur in Notfällen in Anspruch genommen werden. Zentral ist aber, wie gezeigt wird, der Mangel an ökonomischer wie kommunikativer Anschlussfähigkeit, wenn es um die vertragliche Absicherung der Samplenutzung geht.

Dies bedeutet aber keineswegs, dass es sich bei Low-Budget Produktionen um amateurhaft klingende Musik handeln muss. Digitale Aufnahmeund Produktionsmöglichkeiten erlauben es heute mit einem geringen finanziellen Mitteleinsatz äußerst professionell klingende Musik herzustellen. ${ }^{9}$ Zugleich stehen Akteur:innen im Low-Budget Sektor internetbasierte Vermarktungs- und Verkaufsmöglichkeiten zur Verfügung, die im Vergleich zu klassischen Musiklabels nur geringe Einstiegshürden aufweisen. ${ }^{10}$

Das Gros der einschlägigen Plattformen bietet Möglichkeiten, mit hochgeladener Musik Einnahmen zu erzielen. Mit einem gewissen Bekanntheitsgrad sind Low-Budget Musiker:innen somit in der Lage, frei von etablierten Labelstrukturen Musik zu produzieren, zu bewerben, zu verkaufen und durch Abrufe Geld zu verdienen. Diese Unabhängigkeit von etablierten Prozessen der Musikindustrie ist aber nicht allumfassend, sondern stößt an ihre Grenzen, wenn es um die Nutzung fremder Werkteile im Rahmen des Sampling geht. Sampling von Werkteilen kann das Vervielfältigungsrecht sowie das Recht der öffentlichen Zugänglichmachung der Tonträgerhersteller nach $\ 85$ UrhG bzw. Art. 2 lit. c und Art. 3 InfoSoc$\mathrm{RL}^{11}$ berühren. Da es sich bei Samples in der Regel um kleinste Werkteile handelt, denen für sich genommen keine Werkqualität zukommt, spielt hier die Frage der Verletzung der Rechte der Urheber eine untergeordnete Rolle. ${ }^{12}$

9 Smudits, Soziologie der Musikproduktion, in: Gensch/Stöckler/Tschmuck, Musikrezeption, Musikdistribution und Musikproduktion, 262.

$10 \mathrm{Zu}$ nennen sind hier niederschwellige Angebote wie YouTube, SoundCloud, Instagram oder Vimeo. Auch Streaminganbieter wie Spotify, Deezer oder Apple Music sind keineswegs nur professionellen Musiker:innen vorbehalten. Mit geringem Aufwand lassen sich durch Online-Distributionsdienste im Rahmen des SelfPublishing wie Distrokid, Tunecore oder AWAL (Artists Without A Label) eigene Musikwerke veröffentlichen.

11 Richtlinie 2001/29/EG des Europäischen Parlaments und des Rates zur Harmonisierung bestimmter Aspekte des Urheberrechts und der verwandten Schutzrechte in der Informationsgesellschaft vom 22. Mai 2001.

12 Dies mag als Wertungswiderspruch gesehen werden, entstammt aber den unterschiedlichen Schutzzielen der Rechte der Urheber und der Rechte der Tonträgerhersteller. 
Der Einfluss, den die komplexe rechtliche Situation auf die betrachtete Akteur:innengruppe hat, ist allerdings enorm. Die formalrechtliche Absicherung eines Musikstücks entscheidet maßgeblich über seine Verwertbarkeit, seine Publizierbarkeit und dadurch mitunter auch über die ökonomische Situation der Musiker:innen.

\subsection{Sample-Clearing-Ein exkludierendes System}

Da digitales Soundsampling dem Hip-Hop künstlerisch inhärent ist, die Freiheiten, die das Urheberrecht und verwandte Schutzrechte lassen, jedoch eher eng sind, hat sich in der Musikindustrie das Verfahren des Sample-Clearing etabliert, das rechtssichere und umfassend verwertbare Produkte garantieren soll. ${ }^{13}$ Grundsätzlich beschreibt Sample-Clearing einen schlichten Vertragsabschluss über die Einräumung einer Nutzungserlaubnis. Faktisch ist die Zugänglichkeit zu oder Aushandlung von einem solchen Vertrag jedoch eingebettet in komplexe Machtstrukturen. Zumeist liegen die Rechte, insbesondere die Tonträgerherstellerrechte bei den großen oligopolen Musikverlagen (Major-Labels). Innerhalb, aber auch zwischen verschiedenen Verlagen scheint sich mit der zunehmenden Relevanz von Sampling ein System entwickelt zu haben, dass das gegenseitige Sample-Clearing, zumeist gegen Bezahlung, sicherstellt. ${ }^{14}$

Somit besteht mit dem Sample-Clearing theoretisch durchaus eine Möglichkeit, sich der rechtlichen Probleme und Haftungsfragen zu entledigen, die die Nutzung fremder Werksstücke oft mit sich bringt. Sozioökonomisch ist das Verfahren jedoch vielschichtiger und muss im Gesamtkontext der Musikproduktion und deren sozialer Struktur betrachtet werden.

Begreift man die großen Organisationen der Musikindustrie als differenziertes System im Sinne der Theorie funktionaler Differenzierung ${ }^{15}$ wird deutlich, dass sich mit dem internen Differenzierungsgrad der Organisationen der Musikindustrie die Komplexität der Anforderungen an das

13 vgl. Hawkins Music Lett. 2012, 446, 446.

14 Mit Blick auf Erfolgsaussichten und Bekanntheit von Künstler:innen werden Samplenutzungen bisweilen für einen Festpreis, bisweilen für eine Verdienstbeteiligung bzw. Rechteaufteilung über Verwertungsgesellschaften (split) vertraglich zugestanden. Da das Ermitteln der Rechteinhaber:innen nicht immer leicht ist, haben sich im Umfeld der Musikindustrie ganze Sample-Clearing-Agenturen etabliert, die Unterstützung bei der Ermittlung und Klärung von Samples anbieten.

15 vgl. Lubmann, Wirtschaft und Gesellschaft, $50 \mathrm{ff}$. 
Endprodukt erhöht. Große Musiklabels funktionieren arbeitsteilig. Produzent:innen und Musiker:innen verantworten die künstlerisch-technische Dimension des Produktes, das Labelmanagement taxiert die Verdienstziele eines Produktes und reguliert den Mitteleinsatz für die Produktion. Hier werden auch die Preise für Samples ausgehandelt. ${ }^{16}$ Die Rechtsabteilungen erarbeiten entsprechende Vertragswerke und sind für etwaige Haftungsfragen, Regressansprüche oder Klagen zuständig. ${ }^{17}$ Hiermit bedienen Musiklabels die Logiken dreier gesellschaftlicher Subsysteme wie sie in der Luhmann'schen Systemtheorie der Gesellschaft ${ }^{18}$ beschrieben sind: der Kunst, der Wirtschaft und des Rechts. ${ }^{19}$

16 vgl. Hawkins Music Lett. 2012, 446, 446. Siehe hierzu auch Hondros in diesem Band.

17 vgl. Fischer, Urheberrecht und Kreativität, in: Schwetter/Neubauer/Mathei, Musikkulturen, 232; Döhl, Ästhetische Selbstständigkeit, in: Schwetter/Neubauer/ Mathei, Musikkulturen, 277.

18 vgl. Luhmann, Wirtschaft und Gesellschaft; Luhmann, Das Recht der Gesellschaft; Lubmann, Die Kunst der Gesellschaft.

19 Dieser Artikel argumentiert aus einer systemtheoretischen Perspektive, da sich hiermit analytisch sehr sauber die verschiedenen Anforderungen trennen lassen, die den Produkten der Musik entgegengebracht werden. Die Theoriebeschreibung bleibt an dieser Stelle bewusst kursorisch, da es mehr um eine soziologisch und empirisch informierte Analyse der rechtlichen Verfasstheit des Feldes geht als um eine sozialtheoretische. Wie in Abb. 1 gezeigt bestehen zwischen den drei relevanten Subsystemen strukturelle Verbindungen, die sog. strukturellen Koppelungen (vgl. Luhmann, Die Wirtschaft der Gesellschaft, 50 f.). Ausführlicher siehe hierzu Schrör, Organized Creativity Discussion Paper 19/1 2019, 1, 13. 


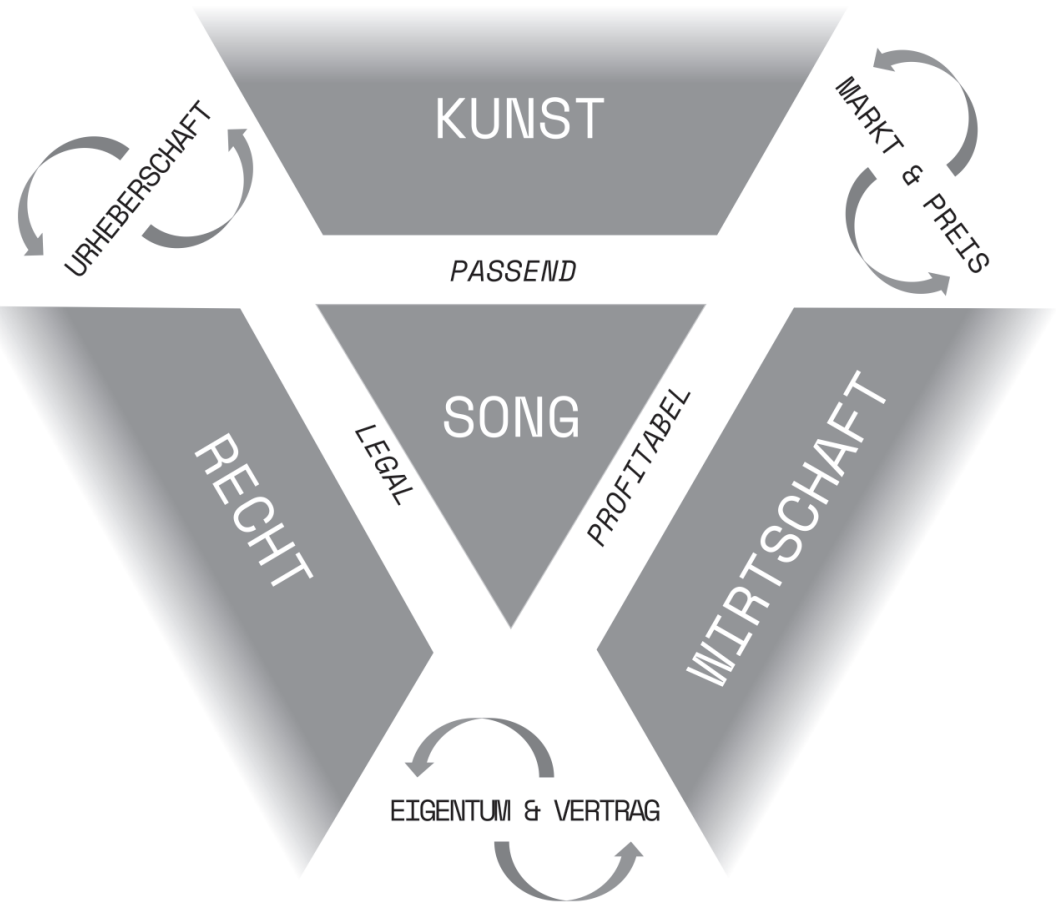

Abb.1: Systemische Anforderungen an ein Musikprodukt

Verwertbare Musikprodukte müssen also eine ganze Reihe systemischer Bedingungen zugleich erfüllen, um erfolgreich zu sein. Sie müssen ein gewisses Maß an künstlerischer Passung ${ }^{20}$ aufweisen, um ein Publikum zu erreichen und zu befriedigen. Bei Hip-Hop Produktionen spielt Sampling eine wichtige Rolle, ist es doch eine selbstverständliche Form des Ausdrucks innerhalb des Genres, die auch von großen, erfolgreichen Künstler:innen vorgelebt wird. Da es sich um ein verwertbares Produkt handelt, muss es wirtschaftlich profitabel sein, also Zahlungen ${ }^{21}$ generieren. Voraussetzung für Funktionalität sind hier möglichst geringe Hürden bei Vermarktung und Platzierung sowie eine grundsätzliche Verwertbarkeit. Diese wiederum ist eng mit der Frage verbunden, ob eine solche Verwertung

21 Luhmann, Die Wirtschaft der Gesellschaft, 52. 
auch rechtlich legal ist ${ }^{22}$, bzw. ob juristische Risiken bestehen, die die Verwertung einschränken können.

Zusammenfassend lässt sich das klassische Sample-Clearing als ein von und in der Musikindustrie etabliertes System begreifen, das sowohl gewisse Rechtskenntnisse und finanzielle Mittel, allem voran aber eine organisationale Einbettung voraussetzt, um an diesem System partizipieren zu können. Aus funktionslogischer Sicht des Kunstsystems ist es zugleich aber ein kulturelles und genretypisches Erfordernis, in Form von Sampling auf die Musik Dritter zurückzugreifen.

Empirische Forschung ${ }^{23}$ indiziert, dass Akteur:innen des Low-Budget Sektors das klassische Sample-Clearing als Verfahren aufgrund der ökonomischen und systemmäßigen Marginalisierung schlicht nicht offensteht. Die Untersuchung illustriert jedoch ebenso, wie sehr Sampling trotz möglicher rechtlicher Probleme eine allgegenwärtige Kulturtechnik ist. Bisweilen werden komplexe Strategien des Verdeckens und Verfremdens angewandt, zum Teil wird auch einfach gehofft, nicht entdeckt zu werden. ${ }^{24}$

Da Low-Budget Musiker:innen sich mit ihrer Musik oft ein Zubrot verdienen, werden ihre Lieder und Alben zumeist auch verwertet. Bei Musiker:innen, die planen, mit ihrer Musik eines Tages ihren gesamten Lebensunterhalt bestreiten zu können, kommt hinzu, dass schon an der Schwelle zum beruflichen Musizieren eine möglichst große Professionalität erreicht werden will und muss. Die bisweilen große technische und musikalische Professionalität, mit der etwa Amateurturniere (sog. Battle, Cypher) auf Plattformen wie YouTube abgehalten werden, führt dazu, dass die ästhetische Produktqualität sich auf den ersten Blick oft kaum von Majorproduktionen unterscheidet.

22 Luhmann, Systemtheorie der Gesellschaft, $467 \mathrm{ff}$.

23 Schrör, Organized Creativity Discussion Paper 19/1 2019.

24 Der Vollständigkeit halber sei an dieser Stelle auf Sample-Datenbanken verwiesen, die meist gegen einen Festpreis, bisweilen aber auch frei verfügbar Samples zur Nutzung anbieten. Solche Samples stehen in einem gewissen Spannungsverhältnis zur beschriebenen Samplingkultur im Hip-Hop. Umfassendere Ausführungen zu solchen präkommodifizierten Soundteilen finden sich bei Hondros in diesem Band. 


\section{Recht als systemisches Gestaltungsinstrument}

Die Analyse des Sample-Clearings aus einer funktionalen Perspektive hat drei wichtige Punkte zutage gebracht. (1) Die Kunstform des Samplings ist im Hip Hop historisch gewachsen und funktional relevant. (2) Die wirtschaftlichen Verwertungslogiken sind vorrangig an Zahlungs- und Verhandlungsmacht gebunden. (3) Das (Urheber-)Recht ist maßgeblich für die empirische Ausgestaltung und die Handlungsmöglichkeiten im Samplingregime. $^{25}$

Besonders letzteres legt nahe, dass es vor allem das Rechtssystem ist, in dem es Stellschrauben gibt, um politisch aktiv auf die Freiheitsgrade in den Subsystemen der Kunst und der Wirtschaft einzuwirken. Die inhärenten Funktionslogiken von Kunst (Streben nach Passung) und Wirtschaft (Streben nach Profit) lassen sich nicht unmittelbar politisch beeinflussen. ${ }^{26}$ Anders ist das bei der regulativen Ausgestaltung, die (neben den autopoietischen Kohärenzbestrebungen des Rechts selbst) immer auch ein politisches Mittel sein kann. ${ }^{27}$

Diese Arbeit verfolgt den Anspruch, Optionen zu eruieren, die zu einem besseren Gleichgewicht im konfliktären systemischen Gefüge beitragen können. Im Folgenden wird deshalb dezidiert auf die Feinheiten und aktuellen Änderungen des formalrechtlichen Regimes im Sampling

25 Die Punkte (1-3) illustrieren den an dieser Stelle nicht weiter ausgearbeiteten Befund, dass neue gesellschaftliche Konfigurationen wie eben eine digitale Kultursphäre von diversen Systemen logisch eingehegt werden, was zu Reibungen, Spannungen und sogar zu weiteren Binnendifferenzierungen der Subsysteme führen kann. Im Sinne einer Verselbstständigung der Systeme erschwert das mitunter die politischen Steuerungspotenziale, besonders in Kunst und Wirtschaft (vgl. Rosewitz/Schimank, Verselbständigung und politische Steuerbarkeit gesellschaftlicher Teilsysteme, in: Mayntz/Rosewitz/Schimank/Stichweh, Differenzierung und Verselbständigung, 295 - 329). Smudits, Soziologie der Musikproduktion, in: Gensch/Stöckler/Tschmuck, Musikrezeption, Musikdistribution und Musikproduktion, 262.

26 Das gilt auch für die Funktionslogik des Rechts, an der sich nichts ändert, wenn die Regulierungen bestimmter Gegenstände angepasst werden.

27 Rosewitz und Schimank merken hierzu an, dass politische Eingriffe in Subsysteme maßgeblich abhängen von „[...]drei politischen Bedingungsfaktoren - politische Steuerungsansprüche, verfügbare politische Steuerungsinstrumente und verfügbares politisches Steuerungswissen[...]“. Das Rechtssystem ist somit im Vergleich zu Kunst und Wirtschaft für politische Steuerung geradezu prädestiniert. (Rosewitz/Schimank Verselbständigung und politische Steuerbarkeit gesellschaftlicher Teilsysteme, in Mayntz/Rosewitz/Schimank/Stichweh, Differenzierung und Verselbständigung, 325, f.). 
eingegangen, um zu prüfen, wie die in Bewegung befindliche Rechtslage das Sampling beeinflusst.

Vor allem die urheberrechtlichen Schrankenregelungen versprechen funktionale Änderungen aus dem beschriebenen systemischen Trilemma des Sample-Clearings. Im Folgenden werden daher die sich vor dem Hintergrund der neueren Rechtsprechung des $\mathrm{EuGH}^{28}$ und des $\mathrm{BGH}^{29} \mathrm{zu}$ fremdreferenzieller Musik abzeichnenden Entwicklungen diskutiert, bevor anhand der Grenzfrage einer etwaigen gewerblichen Nutzung der Blick auf die Richtlinie über das Urheberrecht im digitalen Binnenmarkt (DSM$\mathrm{RL})^{30}$ gerichtet wird.

\subsection{Spezifische Auswirkungen der Metall auf Metall Entscheidungen des EuGH und des $B G H$}

Die rechtliche Lage hinsichtlich der Einordnung von Samples war stets von Unsicherheit geprägt. Bezeichnend hierfür ist der mittlerweile über 20 Jahre andauernde Rechtsstreit Pelham u.a./Hütter u.a. ${ }^{31}$ (Metall auf Metall). Durch die in diesem Verfahren ergangene Entscheidung des EuGH und die neueste Entscheidung des BGH als auch durch die DSM-RL ist Bewegung in die Situation gekommen. Der EuGH stellt in seinem Urteil zu Metall auf Metall fest, dass, sofern das gesampelte Element in dem neuen Werk nicht erkennbar ist, keine Vervielfältigungshandlung angenommen werden kann. ${ }^{32}$ Der BGH führt hierauf aufbauend aus, dass eine Vervielfältigungshandlung gegeben ist, wenn ein durchschnittlicher Musikhörer das

28 EuGH, Urt. v. 29.07.2019 - C-476/17, GRUR 2019, 929 - Metall auf Metall.

29 BGH, Urt. v. 30.04.2020 - I ZR 115/16, GRUR 2020, 843 - Metall auf Metall IV.

30 Richtlinie (EU) 2019/790 des Europäischen Parlaments und des Rates über das Urheberrecht und die verwandten Schutzrechte im digitalen Binnenmarkt vom 17. April 2019.

31 Zuletzt: BGH, Urt. v. 30.04.2020 - I ZR 115/16, GRUR 2020, 843; EuGH, Urt. v. 29.07.2019 - C-476/17, GRUR 2019, 929 - Metall auf Metall; Vorangegangen: LG Hamburg, Urt. v. 08.10.2004 - 308 O 90/99, BeckRS 2013, 7726; OLG Hamburg, Urt. v. 07.06.2006 - 5 U 48/05, GRUR-RR 2007, 3; BGH, Urt. v. 20.11.2008 - I ZR 112/06, GRUR 2009, 403; OLG Hamburg, Urt. v. 17.08.2011 - 5 U 48/05, GRURRR 2011, 396; BGH, Urt. v. 13.12.2012 - I ZR 182/11, GRUR 2013, 614; BVerfG, Urt. v. 31.05.2016 - 1 BVR 1585/13, GRUR 2016, 690; BGH, Vorlagebeschluss v. 01.06.2017 - I ZR 115/16, NJW 2017, 3472.

32 EuGH, Urt. v. 29.07.2019 - C-476/17, GRUR 2019, 929 (Rn. 31) - Metall auf Metall. 
übernommene Fragment im neuen Werk wiedererkennt. ${ }^{33}$ Im konkreten Fall sei dies gegeben. ${ }^{34}$ Der erkennende Senat des Berufungsgerichts habe die entsprechende Stelle bei mehrmaligem Hören beider Stücke wiedererkannt. ${ }^{35}$

\subsubsection{Urheberrechtliche Schranken}

Auch wenn keine vertragliche Vereinbarung über die Nutzung von Werkteilen durch Sampling über das Sample-Clearing erzielt werden kann, so kann die Nutzung dennoch im Rahmen der urheberrechtlichen Schrankenbestimmungen auch ohne die Zustimmung der Rechteinhaber:innen zulässig sein. Im Zusammenhang mit Sampling bislang am bedeutendsten im deutschen Urheberrecht war das Recht der freien Benutzung nach $\mathbb{2} 4$ UrhG. ${ }^{36}$ Der EuGH stellt hierzu fest, dass Art. 5 InfoSoc-RL eine abschlieBende Regelung der Ausnahmen und Beschränkungen der durch das Urheberrecht und die verwandten Schutzrechte gewährten Ausschließlichkeitsrechte trifft. Da Art. 5 InfoSoc-RL ein Recht der freien Benutzung nicht vorsieht, ist dieses nach dem EuGH seit dem Zeitpunkt des Inkrafttretens der InfoSoc-RL nicht mehr mit dem Unionsrecht vereinbar. Auch eine dogmatische Einordnung der freien Benutzung als Bestimmung des Schutzbereichs des Urheberrechts und nicht als Schranke sieht der BGH als nach dem Urteil des EuGH nicht mit dem Unionsrecht vereinbar. ${ }^{37}$ Art. 5 Abs. 3 lit.k InfoSoc-RL sieht vor, dass Mitgliedstaaten Ausnahmen für Karikaturen, Parodien oder Pastiches vorsehen können. Diese Ausnahmen sind im deutschen Urheberrecht nicht explizit vorgesehen. Die Rechtsprechung hat Fälle, die unter Parodie und Karikatur subsumiert werden

33 BGH, Urt. v. 30.04.2020 - I ZR 115/16, GRUR 2020, 843 (Rn. 30 f.) - Metall auf Metall IV.

34 BGH, Urt. v. 30.04.2020 - I ZR 115/16, GRUR 2020, 843 (Rn. 30 f.) - Metall auf Metall IV.

35 BGH, Urt. v. 30.04.2020 - I ZR 115/16, GRUR 2020, 843 (Rn. 30) - Metall auf Metall IV.

36 Der BGH Urt. v. 20.11.2008 - I ZR 112/06, GRUR 2009, 403 (Rn. 21) und das BVerfG Urt. v. 31.05.2016 - 1 BVR 1585/13, GRUR 2016, 690 (Rn. 94) erachten $\$ 24$ UrhG als analog anwendbar auf das im Zusammenhang mit Sampling in der Regel maßgebliche Recht des Tonträgerherstellers.

37 BGH Urt. v. 30.04.2020 - I ZR 115/16, GRUR 2020, 843 (Rn. 32 ff.) - Metall auf Metall IV. 
können, bislang über die freie Benutzung nach $₫ 24$ UrhG gelöst. ${ }^{38}$ Der BGH stellt in seinem Urteil fest, dass die Schranke für Pastiches zugunsten des fraglichen Samples in dem Metall auf Metall- Rechtsstreit nicht in Betracht kommt, da diese Schranke im deutschen Urheberrecht nicht umgesetzt und auch nicht durch die Rechtsprechung anerkannt wurde. ${ }^{39}$ Definiert wird Pastiche als Werk, dessen Stil einem anderen Werk entlehnt ist. $^{40}$ In dem Urteil des BGH liegt die Andeutung, dass im Fall einer Umsetzung der Schranke für Pastiches eine Subsumtion von Samples hierunter in Betracht kommen könnte. Hier sieht der BGH die Gesetzgebung in der Pflicht. ${ }^{41}$

\subsubsection{Sample als Zitat?}

Als Schranke, auf die sampelnde Musiker:innen sich berufen könnten, bleibt das Zitatrecht nach Art. 5 Abs. 3 lit. d InfoSoc-RL, welches über $\$ 51$ UrhG auch im deutschen Urheberrecht vorgesehen ist. Voraussetzung hierfür ist, dass ein besonderer Zweck das Zitat rechtfertigt und auch der Umfang durch den Zweck gerechtfertigt ist. Nach Art. 5 Abs. 3 lit. d InfoSoc-RL muss das Zitat auch den „anständigen Gepflogenheiten“ entsprechen. Zudem müssen Entnahmequelle und Urheber:in des Ursprungswerkes genannt werden, sofern dies nicht unmöglich ist. Diese Voraussetzung entspricht im deutschen Urheberrecht $\$ 63$ UrhG, welcher richtlinienkonform auszulegen ist. ${ }^{42}$

38 BGH Urt. v. 28.7.2016 - I ZR 9/15, GRUR 2016, 1157 (Rn. 28) - „auf fett getrimmt“, die Entscheidung bezieht sich auf Parodien, zu Pastiches gibt es bislang keine Rechtsprechung, zu der Erfassung von Pastiches über $\$ 24$ UrhG: Schulze GRUR 2020, 128, 128.

39 BGH Urt. v. 30.04.2020 - I ZR 115/16, GRUR 2020, 843 (Rn. 65) - Metall auf Metall IV. Karikatur und Parodie erscheinen im Zusammenhang mit Musiksampling abwegig, auch wenn der BGH diese in Rn. 60 ff. diskutiert. Parodie oder Karikatur kämen hier als Schranken grundsätzlich in Betracht, da diese durch die Rechtsprechung bereits vor der Umsetzung der InfoSoc-RL anerkannt waren. Der BGH lehnt diese im Fall aber mit der Begründung ab, es gebe „keinen Anhaltspunkt dafür, dass das Musikstück „Nur mir“ einen Ausdruck von Humor oder eine Verspottung darstell[e]“ (Rn. 63).

40 Zur Definition des Pastiches siehe ausführlich: Pötzlberger GRUR 2018, 675, $677 \mathrm{ff}$.

41 BGH Urt. v. 30.04.2020 - I ZR 115/16, GRUR 2020, 843 (Rn. 65) - Metall auf Metall IV.

42 Leistner GRUR 2019, 1008, 1013; Lüft, in: Wandtke/Bullinger, Praxiskommentar zum Urheberrecht, Vor $\mathbb{S}$ 44a ff. (Rn. 5). 
Als Zitatzweck muss die Übernahme nach dem EuGH der geistigen Auseinandersetzung mit dem Ursprungswerk dienen, was bedeutet, dass eine Interaktion stattfinden muss. ${ }^{43}$ Eine Interaktion kann nach dem EuGH nur bei Erkennbarkeit des übernommenen Werkteils stattfinden. ${ }^{44}$ Der Generalanwalt formuliert in seinem Schlussantrag, hierbei müsse die Fremdheit des übernommenen Werkteils zu erkennen sein. ${ }^{45}$ Hierauf stützt sich der BGH nunmehr. Der BGH sieht es für ein Zitat als notwendig, dass für die Hörer:innen erkennbar ist, dass das übernommene Fragment einem anderen Werk entnommen ist. ${ }^{46}$ Da hierfür im konkreten Fall kein Anhaltspunkt bestehe, lehnt der BGH die Annahme eines Zitates vorliegend ab. ${ }^{47}$ Mit dieser Begründung schließt der BGH allerdings für das Sampling das Zitat als Schranke weitestgehend aus. Eine Herausstellung der übernommenen Fragmente als fremd widerspräche der genretypischen Nutzung. In dem vorangegangenen Urteil des EuGH bleibt hingegen offen, was genau erkennbar bedeutet, ob sich dies auf die Fremdheit des Elements bezieht oder ob es ausreicht, dass bei Kenntnis des Ursprungswerkes das Fragment in dem neuen Werk wiedererkannt werden kann ${ }^{48}$ und aus wessen Sicht dies zu bewerten ist. ${ }^{49}$ Hinsichtlich der Frage aus wessen Sicht dies zu bewerten ist, scheint hier naheliegend auf eine:n Durchschnittshörer:in abzustellen. ${ }^{50}$ Hinsichtlich der Erkennbarkeit ist letztere Interpretation, wonach die Möglichkeit der Wiedererkennung bei einem Vergleich ausreichend ist, samplingfreundlich und den genretypischen Gepflogenheiten entgegenkommend. Gleichzeitig ist bei dieser Interpretation dann fraglich, ob der EuGH einerseits die Übernahme erkennbarer fremder Werkteile, die durch die Zitatschranke gerechtfertigt sein kann, und andererseits eine Nicht-Erkennbarkeit fremder Werkteile, die gar nicht erst

43 EuGH, Urt. v. 29.07.2019 - C-476/17, GRUR 2019, 929 (Rn. 71) - Metall auf Metall.

44 EuGH, Urt. v. 29.07.2019 - C-476/17, GRUR 2019, 929 (Rn. 73) - Metall auf Metall.

45 Szpunar, ZUM 2019, 237 (Rn. 67).

46 BGH, Urt. v. 30.04.2020 - I ZR 115/16, GRUR 2020, 843 (Rn. 54) - Metall auf Metall IV.

47 BGH, Urt. v. 30.04.2020 - I ZR 115/16, GRUR 2020, 843 (Rn. 55) - Metall auf Metall IV.

48 Wobei der BGH erstere Interpretation des EuGH annimmt.

49 Thonemann/Farkas ZUM 2019, 748, 751.

50 Wagner MMR 2019, 727, 729. So auch der BGH, Urt. v. 30.04.2020 - I ZR 115/16, GRUR 2020, 843 (Rn. 29) - Metall auf Metall IV, der hinsichtlich der für die Annahme der Vervielfältigungshandlung erforderlichen Erkennbarkeit auf das „Hörverständnis eines durchschnittlichen Musikhörers“ abstellt. 
als Vervielfältigungshandlung gilt, als „passgenaues Ineinandergreifen“51 zweier Formen der erlaubten Nutzung intendiert. Durchaus zustimmungswürdig vertritt Homar, für eine Interaktion eines Musikstücks mit einem Sample könne bereits die bloße Erkennbarkeit genügen. ${ }^{52}$ Historisch, wie anhand des Genres, scheint dies durchaus begründbar. Anders als die schriftkulturelle Herkunft des Europäischen Urheberrechts ist die moderne Schaffenskultur des Hip-Hop auf deutlich fluidere und weniger formelle Weise entstanden. Das bedeutet jedoch nicht, dass bei fehlender sprachlicher Nennung nicht von einer inhaltlichen Auseinandersetzung gesprochen werden kann. Das direkte ${ }^{53}$ Aufgreifen und Nutzen der Musik Dritter ist als Stilmittel eng mit der Entstehung des Hip-Hop verknüpft. Das hintergründige Abspielen, Mixen und Scratchen von Schallplatten, begleitet von Rap und Gesang ist als Ursprung des Genres zu begreifen und zeigt, wie zentral die Auseinandersetzung, sei sie musikästhetischer oder textlicher Natur, im Hip-Hop ist. Dies entspricht der Wertung des BVerfG, wonach die kunstspezifische Betrachtung gebietet, dass es Künstler:innen offensteht, die Form der künstlerischen Auseinandersetzung frei zu wählen, sowie, dass Spezifika des Genres Berücksichtigung finden. ${ }^{54}$

Die Übernahme übermäßiger Werkteile wird von dem Zitatrecht über die Voraussetzung ausgenommen, dass die Nutzung den „anständigen Gepflogenheiten entsprechen und in ihrem Umfang durch den besonderen Zweck gerechtfertigt “ ${ }^{55}$ sein muss. Nach der Definition des EuGH bedeutet dies, dass „[d]ie Nutzung [...] nicht die Grenzen dessen überschreiten [darf], was zur Erreichung des mit dem fraglichen Zitat verfolgten Ziels erforderlich ist" ${ }^{56}$. Die im Hip-Hop typische Auseinandersetzung mit Fremdmaterial besteht aus der Übernahme kurzer Teile, die zudem in der Regel nicht isoliert stehen, sondern in das neu entstandene Gesamtwerk eingebunden werden. Der Ausschluss langer, isoliert stehender und damit ohnehin untypischer Übernahmen aus der Privilegierung des Zitatrechts würde in diesem Fall der Samplingkultur im Hip-Hop keineswegs widersprechen.

51 Leistner GRUR 2019, 1008, 1012.

52 Homar ZUM 2019, 731, 737.

53 Indirekte Fremdreferenzialität ist musikgeschichtlich noch weiter verbreitet als direkte Samples oder Zitate. Das Orientieren an, das Nutzen oder Ausborgen von Bestehendem zieht sich durch die gesamte Musikgeschichte (vgl. Carroll U. Cin. L. Rev. 2004, 1405; Meconi, Early Musical Borrowing; Carrell, Bach the Borrower).

54 BVerfG, Urt. v. 31.05.2016 - 1 BVR 1585/13, GRUR 2016, 690 (Rn. 99); Leistner GRUR 2019, 1008, 1013.

55 So in Art. 5 Abs. 3 d InfoSoc-RL.

56 EuGH, Urt. v. 29.07.2019 - C-476/17, GRUR 2019, 929 (Rn. 69) - Metall auf Metall. 
Nicht nur die Konkretisierung der Frage der Interaktion mit dem gesampelten Werkteil, auch die Quellenangabe wirft Probleme auf. Der EuGH setzt sich mit der Quellenangabe nicht genauer auseinander. Eine Unmöglichkeit der Quellenangabe kann wohl im sogenannten Micro-Sampling, also dem Sampeln von kleinsten Teilen, angenommen werden. Den Musizierenden scheint oft selbst nicht bewusst, aus welcher Quelle ein Soundschnipsel genau stammt. ${ }^{57} \mathrm{Zu}$ spontan und informell ist das Arrangieren und das kreative Arbeiten mit Studiosoftware und Ordnern voller Musikteilen, die bisweilen bereits in anderen Projekten genutzt und dort mit Effekten verzerrt oder in komplexe Beats eingebaut wurden. Hier schafft die Schwelle der Erkennbarkeit im Unsicherheitsbereich des MicroSamplings zumindest eine gewisse Entspannung. Bei größeren, mitunter offensichtlich erkennbaren Samples kann davon ausgegangen - oder als Sorgfaltspflicht gefordert - werden, dass deren Quellen bekannt sind. Insbesondere bei Verwertungen auf Plattformen steht einer Quellennennung nichts entgegen. ${ }^{58}$

Eine Interpretation der Entscheidung des EuGH dahingehend, dass Sampling unter den oben genannten Voraussetzungen künftig unter die Zitatschranke gefasst werden könnte, scheint dementsprechend mit den kunstspezifischen Anforderungen des Genres vereinbar. Zwar kann nach dem Urteil des BGH der konkrete Fall nicht unter die Zitatschranke subsumiert werden, für die Zukunft deutet sich aber an, dass wohl auch die Pastiche-Schranke in das deutsche Urheberrecht aufgenommen werden muss, sodass Sampling hierunter gefasst werden kann. Für den Ausgangsfall ist dies wenig hilfreich, doch ist die Andeutung der Lösung über eine im deutschen Urheberrecht einzuführende Pastiche-Schranke zu begrüßen, da dies grundsätzlich die Möglichkeit der künstlerischen Interaktion bietet.

\subsection{Auswirkungen der DSM-RL auf die untersuchte Akteur:innengruppe}

Mit der Unsicherheit, der die Akteur:innen ausgesetzt sind, geht die Frage der Haftung für etwaige Rechtsverletzungen einher. Diesbezüglich sind die Auswirkungen der DSM-RL auf sampelnde Musiker:innen zu untersuchen. Art. 17 der DSM-RL führt zu einer Änderung im Haftungsregime

57 Schrör, Organized Creativity Discussion Paper 19/1 2019, 25.

58 Beim Upload von Musikdateien, die verwertbar sein sollen, fällt ohnehin ein gewisser administrativer Aufwand an. Der Mehraufwand einer Nennung im Sinne des Musikzitates gesampelter Werke oder Werksteile in den Metadaten eines Musikuploads erscheint auch praktisch zumutbar. 
hinsichtlich der öffentlichen Wiedergabe und der öffentlichen Zugänglichmachung urheberrechtlich geschützter Werke oder sonstiger Schutzgegenstände. ${ }^{59}$ Eine Rolle spielt dies dann, wenn die Übernahme erkennbar ist und gleichzeitig keine der Schranken des Urheberrechts greift. Die Norm schreibt die Verantwortlichkeit für die öffentliche Wiedergabe und das öffentliche Zugänglichmachen urheberrechtlich geschützter Werke oder sonstiger Schutzgegenstände den jeweiligen Plattformen zu. Offen ist, ob die Haftung der Plattform dazu führt, dass die Haftung der Nutzer:innen entfällt. ${ }^{60}$ An der Haftung der Nutzer:innen für die Vervielfältigungshandlung ändert sich zudem durch die Richtlinie nichts.

\subsection{1 Öffentliche Zugänglichmachung von Werkteilen durch Sampling}

Nach Art. 3 Abs. 2 lit.b InfoSoc-RL und $\$ 85$ Abs. 1 UrhG kommt dem Tonträgerhersteller das ausschließliche Recht $\mathrm{zu}$, zu erlauben oder zu verbieten, dass der Tonträger der Öffentlichkeit zugänglich gemacht wird. ${ }^{61}$ In dem EuGH-Urteil Metall auf Metall ist die Frage des Verstoßes gegen das Recht der öffentlichen Zugänglichmachung nicht streitgegenständlich. Ist ein ein oder mehrere Samples enthaltendes Musikstück aber über das Internet abrufbar, ist dieses jedoch von Relevanz. ${ }^{62}$ Für die betrachtete Gruppe der Sampelnden spielt die Frage der Verletzung des Rechtes der öffentlichen Zugänglichmachung durchaus eine bedeutende Rolle, da diese ihre Werke in der Regel über Plattformen einem Publikum darbieten. Wird ein fremdes Werk in ein Eigenes übernommen, so wird auch Ersteres öffentlich zugänglich gemacht. ${ }^{63}$ Es ist anzunehmen, dass dies entsprechend dem hohen Schutzniveau der InfoSoc-RL und bezogen auf das Recht des Ton-

59 Die DSM- RL greift nach Art. 2 Nr. 6 und Art. 17 Abs. 6 nur für bestimmte Plattformen. Für eine Plattform wie YouTube greift die DSM-RL hiernach eindeutig, für eine Vielzahl anderer Plattformen, die insbesondere auch im Low Budget Bereich von Bedeutung sind, ist dies allerdings fraglich.

60 Dreier GRUR 2019, 771, 776. Nach Hofmann GRUR 2019, 1219, 1228 haften Nutzer:innen weiterhin unabhängig von der Haftung der Plattformen. Faktisch ist wohl zu erwarten, dass vorrangig die Plattform haftbar gemacht wird, da einzelne Nutzer:innen oft schwer zu erreichen sind.

61 Hinsichtlich des Rechts der öffentlichen Wiedergabe verfügt der Tonträgerhersteller über kein Ausschließlichkeitsrecht.

62 Hierzu auch Homar ZUM 2019, 731, $734 \mathrm{f}$.

63 Götting, in: Ahlberg/Götting, BeckOK Urheberrecht, \19a Rn. 5; BGH, Urt. v. 12.11.2009 - I ZR 166/07, MMR 2010, 556 (Rn. 21) - marions-kochbuch.de. 
trägerherstellers auch bei kleinsten Teilen gilt, die von diesem Recht bereits erfasst sind. ${ }^{64}$

\subsubsection{Gewerblichkeit als struktureller Tipping Point}

Nach Art. 17 Abs. 1 DSM-RL soll die Verantwortung für den Abschluss von Lizenzvereinbarungen nunmehr der Plattform obliegen. Durch die Plattform abgeschlossene Lizenzvereinbarungen sollen gleichzeitig auch als Vereinbarung zugunsten der Nutzer:innen der Plattform gelten. ${ }^{65}$ Dies gilt nach Art. 17 Abs. 2 DSM-RL aber nur, „sofern diese Nutzer nicht auf der Grundlage einer gewerblichen Tätigkeit handeln oder mit ihrer Tätigkeit keine erheblichen Einnahmen erzielen". Die beschriebene Gruppe der Low-Budget Musiker:innen bewegt sich im Grenzbereich zur gewerblichen Nutzung. Nach Erwägungsgrund 69 DSM-RL soll die der Plattform erteilte Lizenz nur dann auch Handlungen der Nutzer:innen erfassen, wenn diese Inhalte ohne Gewinnerzielungsabsicht teilen oder „die Gewinne, die die hochgeladenen Inhalte einbringen, im Verhältnis zu den abgedeckten urheberrechtlich relevanten Handlungen der von diesen Erlaubnissen abgedeckten Nutzer nicht erheblich sind“. Nach der Stellungnahme des GRUR Fachausschusses zur Urheberrechtsreform ist entscheidend,

„[...] ob die Handlungen nach ihrem Gesamtbild unter Berücksichtigung sämtlicher Umstände des Einzelfalls typologisch schon der Tätigkeit eines professionellen YouTubers oder Influencers zuzuordnen sind, dessen Dienstleistung auch von einem durchschnittlichen Mitglied seines Besucherkreises nicht mehr als rein private Information zum sozialen Austausch wahrgenommen wird, sondern als professionelle Dienstleistung, die entweder gewerblicher Natur ist oder jedenfalls bewusst der Erzielung erheblicher Einnahmen dienen soll." ${ }^{66}$

Blickt man auf die empirische Situation am Tipping Point des Gewerblichen, so wird deutlich, wie komplex die scheinbare Dichotomie in der Pra-

64 So auch Homar ZUM 2019, 731, $734 \mathrm{f}$. Wobei hier kein rechtspolitisches Argument entwickelt werden soll, vielmehr erfordert die umfassende Analyse der Situation der betrachteten Akteur:innengruppe eine Analyse der geltenden Rechtslage.

$65 \mathrm{Zu}$ beachten ist, dass dies nur gilt, solange der übernommene Teil keine Werkqualität hat. Dem Urheber kommt nach $\$ 23$ UrhG ein Bearbeitungsrecht zu, welches dem Tonträgerhersteller nicht zukommt.

66 Würtenberger/Freischem GRUR 2019, 1140, 1157. 
xis der Musizierenden ist. Das fängt damit an, dass die Gewerblichkeit nur zum Teil in der Entscheidung der Musiker:innen liegt. Bei grundlegendem Verdienstinteresse - welches oft genug nur einen kleinen Zuverdienst erwarten lässt - entscheidet allein der Erfolg der Musik über die erwirtschafteten Gewinne. Virale Dynamiken im Internet ${ }^{67}$ illustrieren sehr gut wie fremdbestimmt Erfolg, gemessen an Aufrufzahlen, sein kann. ${ }^{68}$ Bei Musiker:innen mit einer gewissen Etablierung im Feld und dadurch stabilen, aber nicht erheblichen Einkünften stellt sich die Frage, ob überhaupt der Schritt ins berufliche Musizieren beabsichtigt ist. Zugleich gibt es im HipHop eine gewisse Rekrutierungskultur aus der Amateurszene, in der ein erarbeiteter Aufstieg vom metaphorischen Bordstein zur Skyline ${ }^{69}$ gewürdigt wird.

\subsubsection{Prüfpflichten der Plattformbetreiber}

Die Haftung der Plattformbetreiber entfällt, wenn sie ihren Pflichten aus Art. 17 Abs. 4 DSM-RL nachgekommen sind, also unter anderem nach Abs. 4 lit. $\mathrm{b}$,alle Anstrengungen unternommen haben, um sicherzustellen, dass bestimmte Werke und sonstige Schutzgegenstände, zu denen die Rechteinhaber:innen den Anbietern dieser Dienste einschlägige und notwendige Informationen bereitgestellt haben, nicht verfügbar sind“, was gemeinhin als eine faktische Pflicht zum Einsatz von Filtertechnologien interpretiert wird. ${ }^{70}$ Dies bedeutet aber auch, dass womöglich durch die Plattform eingesetzte (wirksame) Filtertechnologien das Hochladen des entsprechenden Werkes potentiell verhindern könnten. Sampelnde Musi-

67 Etwa die Rapper Soulja Boy, the Weeknd oder Charly Puth, Russ oder Lil Pump, die vor ihrem zum Teil enormen viralen Erfolg bereits längere Zeit Musik auf Plattformen veröffentlichten.

68 Monetarisierung und der damit erhoffte Verdienst müssen keineswegs mit dem Willen oder der Bereitschaft einhergehen, hauptberuflich zu musizieren. Besonders Musiker:innen und Beatproduzent:innen im untersuchten Feld arbeiten oft jahrelang neben einer anderen hauptberuflichen Erwerbstätigkeit oder einer Ausbildung in einem nicht-musikalischen Bereich (vgl. hierzu auch Schrör, Organized Creativity Discussion Paper 19/1 2019, 25 ff.).

69 „Von der Skyline zum Bordstein zurück“ ist der Titel eines Albums des Rappers Bushido. Dieses wird aufgrund von Verstößen gegen das Urheber- und Leistungsschutzrecht, auch im Rahmen des Samplings, nicht mehr vertrieben (LG Hamburg, Urt. v. 23.03.2010 - 310 O 155/08).

70 Spindler CR, 277 (Rn. 53); Specht NJW 2018, 3686, 3689; Pravemann GRUR 2019, 783, 786. 
ker:innen sähen sich somit nicht mehr nur rechtlichen Beschränkungen gegenüber, die in der Praxis des Samplings häufig in der Hoffnung darauf, nicht entdeckt zu werden, ignoriert wurden, sondern Uploads würden gegebenenfalls von vornherein automatisiert verhindert werden. ${ }^{71}$ Die DSMRL manifestiert durch die strengen Prüfpflichten für Plattformbetreiber aber lediglich eine schon seit längerem etablierte Praxis. Aufgrund der Rechtsprechung zu den Prüfpflichten im Rahmen der Störerhaftung ${ }^{72}$ werden seit Jahren Filtertechnologien eingesetzt. ${ }^{73}$ Die rechtliche Lage diesbezüglich hat sich durch die explizite Nennung der Sicherstellung der Schrankenregelungen sowohl für Zitate als auch für Pastiches jedenfalls marginal verbessert. Für sampelnde Musiker:innen, die bereits unter die Definition einer gewerblichen Nutzung fallen, aber nicht bei einem großen Label sind, sodass die Einholung der Lizenz schwierig ist, ergeben sich aus der Urheberrechtsrichtlinie dementsprechend kaum Änderungen. ${ }^{74}$

\section{Fazit}

Bezogen auf die komplexen systemischen Anforderungen, die verwertbare fremdreferenzielle Musikprodukte wie gezeigt erfüllen müssen, kann zwar nur von einer marginalen, doch durchaus von einer als positiv einzuordnenden Verschiebung gesprochen werden. Ohne den Logiken des Wirtschaftssystems und der legitimen Verwertung bestehender Musik zu widersprechen, besteht am temporalen Tipping Point der gegenwärtigen Rechtsentwicklung die Chance, die für künstlerische Funktionalität elementaren Freiheiten und Entfaltungsräume zu stärken. Auch wenn mit dem Urteil des BGH der Rechtsstreit noch nicht abgeschlossen ist, konnten diesem wichtige Erkenntnisse entnommen werden. Das vorangegangene Urteil des EuGH bietet Anlass zur Diskussion der Möglichkeit der Subsumtion von Samples unter das Zitatrecht. Sofern die Schwelle für eine Interaktion

71 Es ist allerdings fraglich, inwieweit Filtertechnologien nach aktuellem technischem Stand dazu in der Lage sind, Samples innerhalb von Stücken zu erkennen.

72 BGH, Urt. v. 12.07.2012 - I ZR 18/11, GRUR 2013, 370 (32 ff.); Urt. v. 15.08.2013 - I ZR 85/12, ZUM-RD 2013, 514 (59 ff.); Urt. v. 30.04.2008 - I ZR 73/05, NJWRR 2008, 1136 (Rn. 53); Urt. v. 19.04.2007 - I ZR 35/04, NJW 2007, 2636 (Rn. 47).

73 So zum Beispiel das Content ID System von YouTube, https://support.google.co $\mathrm{m} /$ youtube/answer/2797370?hl=de, (zuletzt abgerufen am 9.1.2020).

74 Es bleibt freilich die Haftung des Sampelnden, unabhängig von der Frage der Gewerblichkeit, für die Vervielfältigung, sofern weder eine Lizenz erworben wurde noch das Zitatrecht oder eine andere europarechtskonforme Schranke greift. 
mit erkennbarem Fremdmaterial im Rahmen des Zitatrechts niedrig angesetzt wird, böte dies Freiräume für das Sampling. Der BGH sieht jedoch keine der im deutschen Urheberrecht aktuell bestehenden Schranken als einschlägig. Für die Zukunft deutet er aber die Notwendigkeit der Übernahme der Pastiche-Schranke in das deutsche Urheberrecht an. Hier sieht der BGH den Gesetzgeber in der Pflicht. Die DSM-RL führt zu einer Einbeziehung nicht-gewerblich handelnder Akteur:innen in durch Plattformen abgeschlossene Lizenzverträge. Dies dürfte rein rechtlich zu einer Erweiterung der Möglichkeiten des Samplings führen. Eine signifikante weitere Einschränkung sampelnder Musiker:innen durch Filtertechnologien über das bereits bestehende Maß hinaus ist kaum zu erwarten. Im Einzelnen bleibt die Umsetzung der Urheberrechtsrichtlinie abzuwarten. In Gänze weisen die rechtlichen Veränderungen durchaus in Richtung einer vorsichtigen Modernisierung des Samplingregimes, das sowohl für private Hobbymusiker:innen, für Low-Budget Akteur:innen als auch für die GroBen der Branche fairere Bedingungen bietet.

Dieser Beitrag hat zeigen können, wie scheinbar feingranulare Änderungen in der Rechtslage, sei es durch Rechtsprechung oder Rechtsetzung, im interdependenten, funktionalen Gefüge der fremdreferenziellen Musik Änderungen bewirken können. Dem Rechtssystem kommt in diesem Äquilibrium somit eine kritische Rolle zu. Es bietet sich in besonderer Weise an für politische Interventionen, muss jedoch zugleich seine eigene inhärente Funktionalität und Geschlossenheit bewahren ${ }^{75}$. Teubner und Wilke bringen dies wie folgt auf den Punkt:

„Versteht man Politik als gesellschaftsbezogene Intervention in komplexe Systeme, dann ist Recht als Interventionsinstrument nur dann geeignet, wenn es - gemessen an den zu regelnden Problemlagen - adäquat komplex gebaut ist und einen Steuerungsmodus zuläßt, der die horizontale Koordination selbstreferentiell geschlossener Teilsysteme leistet." "76

Die unmittelbare Wirkung, die das Recht auf Kunst und Wirtschaft hat, scheint im kritischen Fall des Samplingregimes den sonst häufig voreiligen Ruf nach dem Gesetzgeber zu legitimieren. Die hier unternommene Darstellung der strukturellen Verbundenheit, der Funktionsweisen und Logi-

75 Es bleibt, wie Luhmann betont, autopoietisch und kommunikativ geschlossen, zugleich aber mit der Gesellschaft verbunden. „Es vollzieht mit jeder Operation auch Gesellschaft [...]. "Luhmann, Das Recht der Gesellschaft, 554.

76 Teubner/Wilke Zeitschrift für Rechtssoziologie 1/1984, 4. 
ken in Recht, Wirtschaft und der Kunst zeigt aber auch, dass es nur geringer Anpassungen bedarf, um manifeste Änderungen im Verhältnis der Funktionssysteme zu erreichen. Etwaige politische Interventionen in das Samplingregime müssen sich zugleich an der Kohärenz und globalen Funktionalität des (Urheber-)Rechts sowie den Wirkungen auf die Kunst und ihrer Verwertungsmechanismen orientieren.

\section{Literatur}

Ablberg, Hartwig/Götting, Horst-Peter (Hrsg.), BeckOK Urheberrecht. 26. Aufl. 2019 [zitiert als: Bearbeiter in: Ahlberg/Götting].

Arewa, Olufunmilayo: From J.C. Bach to Hip Hop: Musical Borrowing, Copyright and Cultural Context, North Carolina Law Review (2006), S. 547-645.

Carrell, Norman, Bach the Borrower, London 1967.

Carroll, Michael: Whose Music Is It Anyway? How We Came to View Musical Expression as a Form of Property, University of Cincinnati Law Review (2004), S. $1405-1496$.

Döhl, Frédéric, Ästhetische Selbstständigkeit als urheberrechtliche Selbstständigkeit. Zur verlorenen Freiheit der freien Benutzung nach $\$ 24$ Abs. 1 UrhG, in: Schwetter, Holger / Neubauer, Hendrik / Mathei, Dennis (Hrsg.), Die Produktivität von Musikkulturen, Wiesbaden 2018, S. 273-288.

Dreier, Thomas: Die Schlacht ist geschlagen - ein Überblick. Zum Ergebnis des Copyright Package der EU-Kommission, GRUR (2019), S. 771.

Fischer, Georg, Die Verwicklung von Urheberrecht und Kreativität in der digitalen Musikproduktion, in: Schwetter, Holger / Neubauer, Hendrik / Mathei, Dennis (Hrsg.), Die Produktivität von Musikkulturen. Wiesbaden 2018, S. 209-243.

Hawkins, Roddy: Review: Creative License: The Law and Culture of Digital Sampling, Music and Letters (2012), S. 446-448.

Hieber, Thomas: "Metall auf Metall" - doch ein Ende ohne Schrecken?, ZUM (2019), S. 746.

Hofmann, Franz: Fünfzehn Thesen zur Plattformhaftung nach Art. 17 der DSM-RL, GRUR (2019), S. 1219.

Homar, Philipp: Enge Handlungsspielräume für das Sampling, ZUM (2019), S. 731.

Kawobl, Friedemann / Kretschmer, Martin, Von Tondichtern und DJs - Urheberrecht zwischen Melodieneigentum und Musikpraxis, in: Hofmann, Jeanette (Hrsg.), Wissen und Eigentum. Geschichte, Recht und Ökonomie stoffloser Güter. Bonn 2006, S. 189-220.

Leistner, Matthias: "Ende gut, alles gut" ... oder "Vorhang zu und alle Fragen offen"? Das salomonische Urteil des EuGH in Sachen "Pelham [Metall auf Metall]", GRUR (2019), S. 1008.

Lubmann, Niklas, Die Wirtschaft der Gesellschaft, Frankfurt a.M. 1994. 
Lubmann, Niklas, Das Recht der Gesellschaft, Frankfurt a.M. 1995.

Lubmann, Niklas, Die Kunst der Gesellschaft, Frankfurt a.M. 1995.

Luhmann, Niklas, Systemtheorie der Gesellschaft (Hrsg. J. F. K. Schmidt \& A. Kieserling), Berlin 2017.

Meconi, Honey, Early Musical Borrowing, New York/London 2004.

Mikos, Lothar, »Interpolation and sampling«: Kulturelles Gedächtnis und Intertextualität im HipHop, in: Androutsopoulos, Jannis (Hrsg.), HipHop. Globale Kultur - lokale Praktiken, Bielefeld 2015, S. 64-84.

Pötzlberger, Florian: Pastiche 2.0: Remixing im Lichte des Unionsrechts - Zu $\$ 24$ UrhG und Art. 5 III k InfoSoc-RL im Kontext der „Metall auf Metall“-Rechtsprechung, GRUR (2018), S. 675.

Pravemann, Timm: Art. 17 der Richtlinie zum Urheberrecht im digitalen Binnenmarkt. Eine Analyse der neuen europäischen Haftungsregelung für Diensteanbieter für das Teilen von Online-Inhalten, GRUR (2019), S. 783.

Rosewitz, Bernd / Schimank, Uwe, Verselbständigung und politische Steuerbarkeit gesellschaftlicher Teilsysteme, in: Mayntz, Renate / Rosewitz, Bernd / Schimank, Uwe / Stichweh, Rudolf (Hrsg.), Differenzierung und Verselbständigung. Zur Entwicklung gesellschaftlicher Teilsysteme. Frankfurt / New York 1988, S. 295 329.

Schrör, Simon, Die Auswirkungen Rechtlicher Unsicherheit Auf Produktionskonventionen in der Low-Budget Musikindustrie, Organized Creativity Discussion Paper 19 no. 1 (2019): S. 1-46, https://www.wiwiss.fu-berlin.de/forschung/organ ized-creativity/media/OC_DiscPapSerie_Simon_Schroer.pdf.

Schulze, Gernot: Die freie Benutzung im Lichte des EuGH-Urteils "Pelham", GRUR (2020), S. 128.

Smudits, Alfred, Soziologie der Musikproduktion, in: Gensch, Gerhard / Stöckler, Eva Maria / Tschmuck, Peter (Hrsg.), Musikrezeption, Musikdistribution und Musikproduktion. Der Wandel des Wertschöpfungsnetzwerks in der Musikwirtschaft, Wiesbaden 2008, S. 241-265.

Specht, Louisa: Die Entwicklung des IT-Rechts im Jahr 2018, NJW (2018), S. 3686.

Spindler, Gerald: Die neue Urheberrechts-Richtlinie der EU, insbesondere „UploadFilter“ - Bittersweet? Analyse der Änderungen beim Text- und Datamining, Leistungsschutz für Presseerzeugnisse und Pflichtenkreis für Hostprovider, CR (2019), S. 277-291.

Stange-Elbe, Joachim / Bronner, Kai, Musikinstrumentenindustrie im digitalen Paradigmenwechsel, in: Gensch, Gerhard / Stöckler, Eva Maria / Tschmuck, Peter (Hrsg.), Musikrezeption, Musikdistribution und Musikproduktion. Der Wandel des Wertschöpfungsnetzwerks in der Musikwirtschaft. Wiesbaden 2008, S. 331334.

Teubner, Gunther /Wilke, Helmut: Kontext und Autonomie: Gesellschaftliche Selbststeuerung durch reflexives Recht, Zeitschrift für Rechtssoziologie (1985/1) S. 435 .

Thonemann, Jannick / Farkas, Thomas: All Samples Cleared? - Oder gehört das Sample "Nur mir", ZUM (2019), S. 748. 
Wagner, Katharina: Entstehung eines neuen Urheberrechtswerks am Beispiel des Musiksampling. Spannungsverhältnis mit vorbestehenden Schutzrechten, MMR (2019), S. 727.

Wandtke, Artur-Axel / Bullinger, Winfried (Hrsg.), Praxiskommentar zum Urheberrecht. 5. Aufl. München 2019 [zitiert als: Bearbeiter in: Wandtke/Bullinger].

Würtenberger, Gert / Freischem, Stephan: Stellungnahme des GRUR-Fachausschusses für Urheber- und Verlagsrecht zur Umsetzung der EU-RL im Urheberrecht (DSM-RL [EU] 2019/790 und Online-SatCab-RL [EU] 2019/789), GRUR (2019), S. 1140. 
